\title{
Cueva, Árbol, Fuente. \\ EL LENGUAJE Y LAS NECESIDADES DEL CUERPO HUMANO EN EL PENSAMIENTO DE ADAM SMITH
}

\author{
CaVE, Tree, Fountain. \\ Language and Human Body NeEds in ADAM SMITH's ThOUght \\ Jorge López Lloret \\ DOI: 10.26754/ojs_arif/a.rif.202013507
}

\begin{abstract}
RESUMEN
Entre 1748 y 1764 Adam Smith definió un proyecto filosófico global y coherente que no llegó a publicar en su integridad. La teoría de los sentimientos morales y La riqueza de las naciones deben interpretarse como partes de este que síllegaron a publicarse. El presente artículo presenta como núcleos vertebradores de dicho proyecto la teoría smithiana del origen del lenguaje y su visión del cuerpo humano como constitutivamente débil y sofisticado. Ambos se hallan en la base del proceso histórico de socialización que, según Smith, fue a la vez, de una manera no problemática, semiótico y económico. Finalmente, reflexiona sobre el interés de esto para la interpretación de Smith como un igualitarista no tan centrado en el mercado y, a partir de aquí, para nuestra visión de nosotros mismos como seres carenciales que deben ampliar sus vínculos sociales todo lo que puedan, pues nos necesitamos.
\end{abstract}

Palabras ClaVE: Adam Smith, Lenguaje, Cuerpo, Economía, Semiótica.

\section{ABSTRACT}

Between 1748 and 1764 Adam Smith defined a global and coherent philosophical project that he failed to publish in its entirety. The Theory of Moral Sentiments and The Wealth of Nations should be interpreted as parts of it that did come to be published. The present article presents as core elements of this project the Smithian theory of the origins of language and his vision of the human body as constitutively weak and sophisticated. Both are at the base of the historical process of socialization that, according to Smith, was together semiotic and economic in a non-problematic way. Finally, it reflects on the interest of this for the interpretation of Smith as an 
egalitarian not so focused on the marked and, from here, for our vision of ourselves as deficient beings that should expand their social ties as much as possible, because we need each other.

KEYwORDS: Adam Smith, Language, Body, Economy, Semiotics.

\section{INTRODUCCIÓN}

Como a la mayoría de los ilustrados escoceses, a Adam Smith le interesaba la naturaleza social del ser humano, en torno a la cual definió un complejo programa de investigación del que La riqueza de las naciones (1776) fue una parte (Ross 2004: 40-59; Griswold 1999: 29-39). Estableció sus líneas generales entre 1748, cuando inició sus conferencias sobre retórica en Edimburgo, y 1764, cuando abandonó su plaza de profesor en la Universidad de Glasgow, aunque murió sin culminarlo. Además de sus dos grandes libros publicados, las obras y documentos más adecuados para reconstruirlo son los que se ubican entre 1748 y 1764, es decir: las Lecciones sobre Jurisprudencia (1762-1763), las Lecciones sobre Retórica (1762-1763) y Consideraciones sobre la formación original de los lenguajes (1761) (Smith 2018; Smith 1985: 201-226). ${ }^{1}$

La lectura aislada de La riqueza de las naciones ha propiciado una visión popular de Smith como el padre del liberalismo moderno, aunque no hace mucho Samuel Fleischacker e Ian McLean, a cuya propuesta nos sumamos, lo presentaron como alguien más bien radical e igualitario (Fleischacker 2005: 72-80; McLean 2006: 120-132). Nuestra intención en este artículo es consolidar esta interpretación recurriendo a la visión smithiana del humano como un ser corporalmente débil y dotado de la capacidad de hablar. Smith no fue especialmente novedoso en esto, aunque sí al sacar de ello unas consecuencias que desarrolló a lo largo de toda su obra, las cuales permiten descubrir su pensamiento como algo más coherente y rico en matices.

En el apartado 2 expondremos la tesis básica del ser humano como un animal corporalmente débil y dotado de la capacidad de hablar. Ensayaremos su potencial hermenéutico afrontando con ella algunos de los temas más recurrentes en la interpretación del pensamiento de Smith. En primer lugar, en el apartado 3 intentaremos apuntalar la tesis de quienes creen que no hay un "problema de

\footnotetext{
1 Citaremos las ediciones en castellano de las obras de Smith. También proporcionaremos la referencia de la edición inglesa (la Glasgow Edition, en la versión de la editorial Liberty).
} 
Adam Smith". Esto nos conducirá, en el apartado 4, a descartar una interpretación teológica de la metáfora de la "mano invisible". Finalmente, en el apartado 5 concluiremos que los dos puntos anteriores niegan cualquier visión del humano como un ser corrupto o moralmente débil, apuntando con ello a las consecuencias que esta visión del pensamiento de Smith podría tener para la manera en la que afrontamos nuestra vida colectiva.

\section{LA NECESIDAD CORPORAL Y EL LENGUAJE}

Según Dugald Stewart, Smith fue un maestro de la historia conjetural (Smith 1998: 252; Smith 1982c: 293), consistente en la reconstrucción hipotética de aquellos hechos del pasado a los que no teníamos acceso. Smith contaba con los precedentes de la historia del lenguaje de Condillac (Condillac 1999: 152-162) y la descripción de la vida primitiva de Rousseau, quien dijo que solo aventuró algunas conjeturas sobre algo "que ya no existe, que quizá no ha existido, que probablemente no existirá jamás” (Rousseau 1989: 111). En cualquier caso, estas conjeturas importan menos por la luz que arrojan sobre el pasado humano que por el proyecto filosófico y social que trataron de justificar, al que nos referiremos en el siguiente apartado.

La historia conjetural de Smith se materializó sobre todo en su escrito Consideraciones sobre la formación original de los lenguajes (Bascones y Domínguez 2011: 60). Aunque se refirió básicamente a la palabra hablada, resulta difícil creer que se limitara a ella, máxime si tenemos en cuenta su dependencia con respecto al Ensayo sobre el origen de los conocimientos humanos de Condillac, quien argumentó a partir de un ejemplo que Smith daría por conocido: un niño hambriento que se esforzaba por coger la fruta de un árbol y otro que lo ayudaba. Condillac se esforzó por mostrar que el surgimiento del signo lingüístico permitió estabilizar la relación entre el estado del sujeto (hambre) y el objeto con el que se vinculaba (fruta) en una situación cooperativa. No conjeturó una génesis intencional sino un encuentro contingente entre dos sujetos, uno de los cuales, el actor, escenificó involuntaria, aunque ostensiblemente, sus necesidades gritando y moviendo su cuerpo hacia el objeto deseado (Condillac 1999: 153). Si por casualidad el espectador pasara por allí, repararía tanto en sus movimientos y sonidos como en el objeto al que apuntaban, impulsándolo su instinto a ayudar. Esto enseñó al actor a conectar los movimientos de su cuerpo con el objeto deseado y con el espectador altruista, institucionalizándose el lenguaje con toda su complejidad gestual, de donde se acabó desgajando la palabra hablada (Wells 1987: 8-18). Esta situación es la que subyace al inicio de Consideraciones. 
Según Smith, "la designación de nombres particulares para denotar objetos particulares, es decir, el establecimiento de los sustantivos, fue probablemente uno de los primeros pasos hacia la formación del lenguaje" (Smith 2018: 39; Smith 1985: 101). Aunque se puede hacer una lectura denotativa y racionalista de esta afirmación, creemos que lo que siguió diciendo lo impide:

Dos salvajes criados lejos de las sociedades humanas y a los que nunca se ha enseñado a hablar, comenzarían naturalmente a formar aquel lenguaje con el que intentarían hacer inteligibles el uno al otro sus necesidades comunes, pronunciando ciertos sonidos siempre que pretendían denotar ciertos objetos. Solo asignarían nombres particulares a los objetos que les eran más familiares y tenían ocasión más frecuente de mencionar (Smith 2018: 39-40; Smith 1985: 203).

En esta primera conjetura de Smith es importante que las necesidades fueran mutuas, pues el fundamento del lenguaje era el mismo que el de la socialización colaborativa, a saber, la urgencia que tenían dos seres por exponer sus carencias para obtener ayuda y ayudar. Creemos que Smith comenzó con los nombres y el lenguaje hablado por abreviar, pero que concordaría con el modelo francés de Condillac y Rousseau, en el que el lenguaje hablado surgió como un subsistema en el proceso de semiotización integral del cuerpo humano.

En segundo lugar, resulta de gran interés la conjetura de Smith sobre las primeras palabras que posiblemente se emitieron, pues introduce otro matiz de naturaleza corporal:

La cueva particular cuyo abrigo los protegía del mal tiempo, el árbol particular cuyo fruto aliviaba su hambre o la fuente particular cuya agua calmaba su sed serían denominados en primer lugar cueva, árbol y fuente o con cualquier otra denominación que, con aquella jerga primitiva, pudieran considerar adecuada para señalarlos" (Smith 2018: 40; Smith 1985: 203).

Las posibles condiciones de subsistencia de la vida original en los bosques tuvieron gran importancia para Rousseau y Condillac (Rousseau 1989: 122; Condillac 1999: 153), a lo que seguramente se debe el que Smith pensase en la palabra "árbol" como una de las primeras, simbolizando la comida que paliaba el hambre. La cueva representaba el abrigo ante el desamparo y con ambas se recogían los dos tipos de necesidades básicas: las del cuerpo interior y las del cuerpo exterior, ambas bases naturales de nuestra debilidad.

Smith abundó en eso en las Lecciones sobre Jurisprudencia que impartió durante el Curso 1762-1763, parte de las cuales nos han llegado como apuntes de alumnos. Estas lecciones son estrictamente contemporáneas de Consideraciones, de las 
Lecciones sobre Retórica, de La teoría de los sentimientos morales y del Borrador de La riqueza de las naciones, es decir, pertenecen a un periodo nodular en el desarrollo del proyecto smithiano. En ellas describió la condición humana de una manera complementaria a la conjetura de Consideraciones:

El hombre ha recibido de la generosidad de la naturaleza razón e ingenio, arte, invención y una capacidad de mejora muy superior a la que ha otorgado a cualquiera de los otros animales; pero está, al mismo tiempo, en una condición mucho más desvalida y necesitada con respecto al mantenimiento y la comodidad de su vida. Todos los otros animales encuentran su comida en el estado en que la desean y la que mejor les viene a sus distintas naturalezas, y pocas otras necesidades tienen. Pero el hombre, de una condición más delicada y una constitución más débil, no se encuentra con nada tan adaptado a su uso que no necesite mejora y preparación (Smith 1995: 380; Smith 1982b: 334).

Se trata de una matriz estructural en la que la debilidad y la inteligencia se requieren y refuerzan entre sí, pues seríamos inviables sin la inteligencia, que sería innecesaria sin la debilidad. Otros dos ingredientes, la delicadeza de nuestro cuerpo y la imaginación de nuestra mente, explican que las soluciones inteligentes a la debilidad sean diversas, comenzando por las necesidades de cocinar los alimentos (pues nuestro estómago es delicado) y confeccionar las ropas (pues nuestra piel es sensible). Por ejemplo, según Smith, la piel humana, a diferencia de la de otros animales, no puede adaptarse a su entorno, lo que explica el desarrollo de la ropa y la arquitectura, con las que busca crear "alrededor de su cuerpo una especie de nueva atmósfera, más suave, cálida y confortable que la del aire circundante común" (Smith 1995: 381; Smith 1982b: 334-335). El ser humano, débil y delicado, pero también inteligente e imaginativo, se pudo hacer un clima a su medida. En principio, esto se podía desarrollar de una manera individual y Smith, de hecho, recogió las conjeturas de Rousseau y Laugier (Rousseau 1989: 163 y 171; Laugier 1999: 44-45) cuando afirmó que el individuo podía afrontar estas necesidades, pues "unas pocas pieles, cosidas quizás con unas pocas tiras de lo mismo, le proporcionan vestimenta; y unos pocos palos clavados en el suelo y cubiertos con pieles o matas le facilitan un refugio" (Smith 1995: 381; Smith 1982b: 335). No obstante, el lenguaje permitiría que todo esto se afrontara mejor.

La conjetura de que el lenguaje, por su naturaleza colaborativa, permitía superar las desventajas corporales del ser humano, provenía de la retórica clásica, que Smith conocía bien. Por ejemplo, Cicerón afirmó en La invención retórica que, aunque "los hombres son en muchos aspectos inferiores y más débiles que los animales, los superan especialmente por la capacidad de hablar" (Cicerón 1997: 91). Smith 
usó esta afirmación contra la conjetura de Rousseau, quien seguramente también la conocía, pues parte de su originalidad consistió en el rechazo de este argumento, afirmando tanto la suficiencia corporal del ser humano original como la inutilidad del lenguaje en este estado (Rousseau 1989: 122-123). Rousseau no afirmaba que el ser humano fuera social y dispusiera de lenguaje por ser débil, sino que llegó a ser débil desde que fue social. Previamente, su cuerpo individual estaba adaptado y era viable, con lo que el lenguaje y la sociedad resultaban innecesarios (Rousseau 1989: 123 y 138). Smith admiró el Segundo Discurso de Rousseau, como mostró en su segunda colaboración con la Edinburgh Review (1756) (Smith 1998: 220-225; Smith 1982c: 250-256), pero al afirmar sin ambages tanto la debilidad del cuerpo humano como su capacidad para el lenguaje recurrió a una conjetura clásica para rechazar directamente la propuesta roussoniana (Griswold 2018: 93-149).

Smith identificó a Rousseau con Mandeville, en el sentido de que "ambos suponen que no existe en el ser humano ningún instinto poderoso que necesariamente lo impulse a buscar la sociedad" (Smith 1998: 220; Smith 1982c: 250; Douglass 2017: 597-620). Era una idea que sintonizaba con la conjetura hobbesiana de un estado de naturaleza brutal. Ante este caso el recurso a Cicerón no servía, pues este aceptaba la brutalidad del estado de naturaleza previo a la implantación de la palabra (Cicerón 1997: 87-88). Smith pensaba que dicha brutalidad pertenecía a la naturaleza (Smith 1998: 59; Smith 1982c: 48), no a las personas. Más próximo a Rousseau en esto, creía en la sensibilidad natural humana ante los sufrimientos de los otros, algo propuesto por su maestro Francis Hutcheson (desde su rechazo de la teoría de la risa de Hobbes; Hutcheson 1750: 26-38) y que sintonizaba con Condillac y Hume, dos de sus referentes. De hecho, Condillac conectó la institucionalización del lenguaje con la sensibilidad natural ante el sufrimiento ajeno:

El otro, conmovido por este espectáculo, fijaba los ojos en el mismo objeto; y, sintiendo pasar a su alma los sentimientos de los que aún era incapaz de dar razón, sufría al ver sufrir a ese desgraciado. Desde ese momento, siéntese interesado en aliviarle, y obedece a esta impresión tanto cuanto le es posible (Condorcet 1999: 153-154).

Al referirse a la simpatía, Hume explicó esto como una inclinación mediante la que una idea se convertía, primero, en una impresión y, segundo, en una pasión (Hume 1988: 439-440). Smith aceptaba esto con unas matizaciones a las que después nos referiremos.

Por lo tanto, recurriendo al papel socializador que Cicerón concedió al lenguaje y a las ideas de Condillac y Hume sobre la empatía innata del ser humano 
hacia sus semejantes, Smith elaboró una conjetura que se oponía a la de Rousseau y cuya originalidad residía en la creencia de que el desarrollo económico no abolía la naturaleza carencial y delicada del cuerpo humano. Aunque a partir de cierto momento, gracias al lenguaje, la subsistencia dejaba de ser precaria (Smith 1998: 61; Smith 1982c: 50), eso no desvinculó a las personas de su condición carencial, como mostró Gloria Vivenza (Vivenza 2001: 19).

Podemos definir a Smith como un progresista matizado. No rechazó en general el progreso humano, como Rousseau, ni se manifestó claramente con respecto a la legitimidad de la expansión colonialista europea, como Diderot, quien la puso en duda (Diderot 2013: 29-30; Bury 2009: 184-198). Aceptó sin más que la ciencia, la técnica y la economía (en un sentido cuantitativo) progresaban, pero no que lo hiciera nuestro cuerpo carencial, de modo que no había ninguna diferencia entre los humanos que habitaban el mundo comercial y aquellos con los que abrió Consideraciones, a cuya debilidad corporal y habilidad lingüística se refirió en las Lecciones sobre Jurisprudencia. Aunque pensaba que el lenguaje y la sociedad colaborativa permitieron que nuestra imaginación afrontara nuestra condición de una manera cada vez más superflua, creando nuevas necesidades (un diagnóstico con claros matices roussonianos; véase Rasmussen 2008: 51-90), sus bases seguían siendo "las necesidades de comer, beber, vestirse y alojarse" (Smith 1995: 383; Smith 1982b: 337), es decir, nuestras carencias corporales. En las sociedades comerciales las palabras fundamentales seguían siendo "cueva", "árbol" y "fuente" y en su opulencia el desamparo, el hambre y la sed no dejaban de acechar, aunque de formas insospechadas y prescindibles. Coincidiría, pues, con la respuesta negativa que dio Diderot a la pregunta de si la condición del ser humano original era "peor que la del ser maravilloso que elige un colchón de plumas para acostarse, que teje el hilo del gusano de seda para vestirse, que ha convertido la caverna, que fue su primera morada, en un palacio, y que ha sabido dar una variedad infinita a sus comodidades y sus necesidades" (Diderot 2011: 84).

En ocasiones Smith incluso identificó procesos de decadencia. Su teoría de las consecuencias impremeditadas de nuestras decisiones y acciones, que trataremos en el siguiente apartado, también discurrió desde lo mejor hasta lo peor. Pensemos, por ejemplo, en los efectos negativos de la división del trabajo, frente a los que reclamó un intervencionismo educativo (Smith 1981: 783-786); o en el desarrollo de la vanidad y la superficialidad en las sociedades comerciales como efecto de la loable simpatía (Smith 1997: 325-336; Smith 1982a: 179-187); o en la lamentable pérdida de la capacidad creadora del lenguaje a medida que se fue definiendo la sintaxis moderna (Smith 2018: 94-101; Smith 1985: 224-226). Si 
comparamos su descripción de los obreros divididos con la de los participantes en las celebraciones colectivas de las que surgió la música, en una época menos mediatizada por el trabajo y el consumo, en la que las necesidades del cuerpo, siendo las mismas, se satisfacían de una manera menos costosa (Smith: 1985: 137; Smith 1998: 186; Smith 1982c: 189), aceptaremos que su pensamiento estaba más allá (o más acá) del progreso y el retroceso.

\section{LA MANO INVISIBLE Y EL CUERPO QUE HABLA}

Según la conjetura de Smith, la situación comunicativa original surgió como una respuesta a nuestras carencias. Con esto se puso en marcha un mundo semiótico que, a través de nuestra imaginación, prolongó la debilidad de nuestro cuerpo en la ropa, la arquitectura y la comida elaborada. Estas extensiones se fueron codificando de una manera cada vez más minuciosa, hasta volverse disciplinas artísticas (Smith 1997: 326; Smith 1982a: 179-180), pero nunca abandonaron sus conexiones con nuestro cuerpo. Por ejemplo, pese a todo su boato y cuidado formal, los palacios de los ricos tan solo tienden "a proteger del sol y la lluvia, de la necesidad y la fatiga" (Smith 1997: 335; Smith 1982a: 186). No eran, pues, sino una variedad de la cueva o la cabaña primitiva, algo que protegía del sol y la lluvia, es decir, que seguía respondiendo a las mismas necesidades del delicado cuerpo humano, aunque fuera una respuesta hinchada.

La peculiaridad de la arquitectura era su potencial de desarrollo semiótico, mayor que el de otras artes que, como las de la comida o la bebida, respondían a diferentes necesidades corporales. Así, Smith afirmaba que ningún terrateniente pensaría en consumir toda su cosecha, pues "la capacidad de su estómago no guarda proporción alguna con la inmensidad de sus deseos, y no recibirá más que el más modesto de los campesinos" (Smith 1997: 332; Smith 1982a: 184). Introdujo en este contexto por primera vez la metáfora de la mano invisible en un sentido económico (Schliesser 2017: 238-245; Samuels 2014: 60-82), por lo que suele leerse en conexión con el uso de la misma metáfora en La riqueza de las naciones (Kennedy 2011: 53-57; Fleischacker 2006: 138-142), aunque parte de su lógica reside en que se lea en conexión con el párrafo sobre la arquitectura, pues la redistribución de la riqueza, producida como por obra de una "mano invisible", se basaba en la diferenciación entre dos tipos de desarrollo, el que parte de las limitadas necesidades interiores (la comida y la bebida) y el que lo hace de las menos limitadas exteriores, como la ropa y la arquitectura. Era la fijación del rico con estos signos más desarrollables lo que permitía la distribución entre los demás 
de lo que se relacionaba con aquellas necesidades cuya limitación las hacía menos significativas. La concentración en la riqueza relacionada con la "cueva" permitía satisfacer más democráticamente las necesidades relacionadas con el "árbol” y la "fuente".

Smith fue explícito al hacer depender todo el desarrollo semiótico de la arquitectura de la debilidad y el refinamiento de la piel humana, afirmando que el poder y la riqueza no eran más que "unas máquinas enormes y laboriosas preparadas para producir unas insignificantes conveniencias para el cuerpo" (Smith 1997: 330; Smith 1982a: 182-183). El guardarropa y el palacio del rico eran, como las pieles y la choza originales, una respuesta a las necesidades de cobijo y amparo, las cuales, al contrario que la comida y la bebida, pudieron diversificarse más y, con ello, resultar más significativas. La ropa y los muebles a la moda, el salón decorado o el jardín paisajístico, en tanto que desarrollos de nuestra piel débil y sofisticada, prolongaban la semiótica gestual que se puso en marcha cuando un ser humano, delante de otros, escenificó su esfuerzo para obtener ayuda y ayudar, pese a que su desmesura condujera a la vanidad (Conlin 2016: 83-85; Fleischacker 2006: 115118). Era el precio que pagar para redistribuir la riqueza.

Puesto que Smith fue muy preciso en la construcción de sus argumentos y la selección de sus ejemplos, es significativo que explicase la diferenciación de los oficios (paso previo a la división del trabajo) recurriendo a la ropa, una de las extensiones de la piel humana. Para realzar la naturaleza cooperativa del mundo comercial moderno comparó, de una manera muy roussoniana, las prendas que cada ser humano, en la primera época económica (la de la caza), confeccionaría para sí mismo con unas pocas pieles, con "la chaqueta de lana que abriga al jornalero" moderno (Smith 1995: 381; Smith 1982b). En un párrafo trepidante, enumeró el alto número de profesionales implicados en la fabricación de esta prenda (Smith 1995: 385-386; Smith 1982b: 338-339; Smith 1994: 41-42; Smith 1981: 22-24). En torno a nuestra piel se fue tejiendo una tupida red de oficios interconectados que alcanzó su mayor desmesura en la era comercial. Además, todos ellos se unían entre sí gracias a nuestra tendencia al trueque, fundamentada en el lenguaje (Smith 1994: 44; Smith 1981: 25). Smith recurrió a la poca pretenciosa chaqueta de un jornalero, de modo que resultara evidente que en los superfluos vestidos a la moda de su época, cada vez más trabajados y artísticos (Ashelford 1996: 121-166), la situación era aún más compleja. Este proceso se basaba en el potencial semiótico de las extensiones de la piel humana, que al marcar con más fuerza el estatus lograban captar más fácilmente la simpatía de los demás y, con ella, su disposición a ayudarnos. 
Smith extendió esto a la parte más famosa de La riqueza de las naciones, la división interior de cada profesión. Su conocido ejemplo de la división de la fabricación de alfileres en dieciocho operaciones provenía de la Enciclopedia de Diderot y d'Alembert (Smith 1994: 34-35; Smith 1981: 14-15 Mankin 2008: 181-205). En la entrada "Epingle" se describía su fabricación y se enumeraban sus tipos: alfileres para hacer calzas, paños, prendas, etc. (Diderot y d'Alembert 1751: 806). Era básicamente un instrumento para la confección de la segunda piel que necesitábamos formarnos. La división del trabajo en este ejemplo, como en casi todos los demás, era parte de la respuesta colectiva al desamparo. Los ejemplos de Smith formaban un sistema que mostraba la complejidad del vínculo social que se anudó en torno a la debilidad y la delicadeza del cuerpo humano, el cual, con la ayuda de los demás permitida por el lenguaje, pudo construirse un entorno artificial protector, coherente y poderosamente semiótico.

En La riqueza de las naciones, pues, las necesidades primordiales seguían constituyendo el eje del proceso social y económico, por muy sofisticado que llegara a ser en su etapa comercial. Cuando Smith afirmó que "después de la comida, las dos grandes necesidades de la humanidad son el vestido y la vivienda" (Smith 1994: 232; Smith 1981: 178), vinculó claramente dicho proceso con las distintas necesidades de nuestro cuerpo, es decir, con el primer párrafo de Consideraciones. No fue accidental que aquí enumerase primero las necesidades de comer y beber ("árbol" y "fuente") y después la de amparo ("cueva"), pues la aplicación de la imaginación a cada caso dependía de sus posibilidades diferentes de diversificación y crecimiento.

Smith caracterizó cada época económica a partir de la proporción de los recursos utilizados para comer y beber, por un lado, y para cobijarse, por otro. Así, con la desafortunada (y actualmente inaceptable) terminología de su época, afirmó que "entre las naciones salvajes y bárbaras, una centésima parte del trabajo anual bastará para proveerlas del vestido y el alojamiento satisfactorios para la mayoría de los habitantes. Las otras noventa y nueve partes resultan a menudo apenas suficientes para proveerlas de comida" (Smith 1994: 235; Smith 1981: 180). Al principio el "árbol" y la "fuente" (el hambre y la sed) eran social y económicamente más importantes que la "cueva" (el desamparo), definiendo una relación social más igualitaria porque su diferenciación económica y semiótica era menor, pero en las sociedades comerciales la importancia relativa de cada ámbito se invirtió, siendo más lo destinado a la ropa y la vivienda que a la comida y la bebida. Smith sintetizó esto así: 
Cuando gracias a la roturación y el cultivo de la tierra, el trabajo de una familia puede suministrar comida para dos, el trabajo de la mitad de la sociedad resulta suficiente para obtener alimento para el conjunto. La otra mitad [...] puede ser empleada en suministrar otras cosas, o en satisfacer las otras necesidades y caprichos de la humanidad. El atuendo y la vivienda, los muebles y lo que llaman el equipo, son los objetos principales del grueso de esas necesidades y caprichos. El rico no consume más comida que su vecino pobre [...] Pero al comparar el amplio palacio y el surtido guardarropa de uno con la choza y los harapos del otro se comprende que la diferencia entre su indumentaria, alojamiento y mobiliario es casi tan grande en cantidad como en calidad. El apetito de alimentos está limitado en cada persona por la estrecha capacidad del estómago humano, pero el afán de comodidades y adornos en la casa, el vestido, el mobiliario y el equipo no parece tener límites ni conocer fronteras (Smith 1994: 235-236; Smith 1981: 180-181).

A partir de las necesidades de un cuerpo débil y delicado, afrontadas cooperativamente gracias al lenguaje, Smith extendió sus argumentos tanto a los mínimos (y máximos) entresijos de la economía como al potencial semiótico y comunicativo de la cultura (Smith 1995: 384; Smith 1982b: 338). Los objetos culturales, como los artísticos, no estaban desvinculados de las necesidades corporales, sino que eran su resultado final, volviéndose superfluos y, con ello, significativamente puros, como Smith planteó en sus análisis del lenguaje poético y la música instrumental (Smith 1985: 117; Smith 1998: 203-204; Smith 1982c: 206-207; Seidel 1989: 500).

En gran medida, nuestro análisis en este apartado se ha basado en el párrafo de La teoría de los sentimientos morales en el que Smith recurrió a la metáfora de la mano invisible, lo que nos conduce a la misma tal y como aparece en La riqueza de las naciones. Aunque se trata de un tema muy especial y sobre el que existe una ingente literatura (Kennedy 2009: 239-263). La interpretación de metáfora de la mano invisible desde la Teología Natural, afirmando la participación de un Dios benigno que saca beneficios colectivos del egoísmo privado, se basa en la conexión de la afirmación de Smith de que cuando comerciamos con los demás no apelamos a su humanidad sino a su interés privado (Smith 1994: 46; Smith 1981: 27) y el párrafo en el que aparece la metáfora (Smith 1994: 554; Smith 1981: 456), argumentalmente distante de aquella y que afronta un problema diferente, a saber, el del efecto negativo de las medidas políticas que obstaculicen el libre comercio de las mercancías. El argumento del segundo texto no se basaba en el egoísmo sino en la ignorancia y, a su vez, en el primero el egoísmo no era tanto una condición antropológica de los agentes, cuanto un tópico argumental de los persuasores, un recurso retórico. 
La mano invisible puede interpretarse, pues, como un argumento de Teología Natural o como un recurso retórico. Aboga por lo primero el que Smith perteneciera a una sólida tradición intelectual en la que la Teología Natural proponía la existencia de un Diseñador inteligente del mundo, incluida la sociedad humana. La importancia de este concepto en el siglo XVII fue muy grande (la aceptaron John Ray, Richard Bentley e Isaac Newton, entre otros) y fue heredado por muchos durante el siglo XVIII, desde Bernard Nieuwentyt hasta, a comienzos del siglo XIX, William Paley (Jantzen 2014: 71-133). El maestro de Smith, Francis Hutcheson, desarrolló con solidez este argumento, afirmando que Dios había diseñado tanto los seres organizados como la benéfica adaptación a sus condiciones de existencia (Hutcheson 2008: 47; Hutcheson 2006: 174-175), lo que incluía al ser humano como agente social, tal y como afirmó:

Todo aquel que crea que este universo, particularmente la naturaleza humana, fue formado por la sabiduría y deliberación de una Deidad, debe esperar encontrar en nuestra estructura evidencias claras que muestren la tarea propia del ser humano, el tipo de vida y las ocupaciones para las que nos ha formado la providencia y sabiduría de nuestro Creador (Hutcheson 2007: 24; traducción nuestra).

Recurriendo a argumentos que provenían del cálculo de probabilidades, Hutcheson afirmaba que podría haber una infinidad de casos en los que la suma de felicidad superase a la de miseria y que nuestra suerte era "absolutamente buena, pese a que contenía una considerable mezcla de mal" (Hutcheson 2002: 43; traducción nuestra). Smith conocía que Hutcheson propuso que el mundo no se podía autoorganizar, sino que lo que en él sucedía, incluida la situación natural y social del ser humano, fue algo diseñado por Dios, más allá de nuestra propia participación. De hecho, sobre todo en La teoría de los sentimientos morales usó en ocasiones un lenguaje que recuerda al de la Teología Natural (Kennedy 2017: 190-193), lo que ha llevado a pensar que este es el contexto adecuado para interpretar la metáfora de la mano invisible (Oslington 2014: 63). Por ejemplo, cuando escribió que "la administración del gran sistema de universo, el cuidado de la felicidad universal de todos los seres racionales y sensibles, es la labor de Dios, no del hombre" (Smith 1997: Smith 1982a: 237), adoptó un tono claramente teológico natural. Si atendemos al contexto argumental, sin embargo, lo que Smith está diciendo es que la economía, incluidas sus consecuencias, no es tarea divina sino humana (Griswold 1999: 141). Además, si Smith hubiera tenido en mente la agencia de Dios, lo peor hubiera sido recurrir a una referencia tan confusa como la de una mano invisible, en lugar de mencionar, simplemente, la agencia de Dios. 
Por otra parte, Smith recurrió a la palabra "Naturaleza" en más ocasiones que a la palabra "Dios". Su uso del término se corresponde con el tercero de los sentidos en los que, según Arthur O. Lovejoy, se ha utilizado: "el orden cósmico como un todo o como un poder cuasi personal (natura naturans) manifestado a su través" (Lovejoy 1952: 72). También podría tratarse, por otra parte, de algo más secular, de lo que Basil Willey, refiriéndose a Hume, definió como un "hábito de la mente" (Willey 1950: 110).

Smith fue discípulo de Hutcheson, pero también admiraba a la segunda generación de ilustrados franceses, entre ellos Diderot. Hutcheson afirmó que Dios hizo tanto a las especies animales como a los medios a los que se adaptaba cada una, pero Diderot afirmó que, si "se llena un amplio terreno de escombros lanzados al azar", entre ellos "los gusanos y las hormigas encuentran hogares muy cómodos" (Diderot 2016: 65). Smith no pudo conocer estas palabras de Diderot, que se publicaron póstumamente, pero sí la filosofía natural que les subyacía, publicada en 1754 en Sobre la interpretación de la naturaleza. El concepto fundamental era el de autoorganización, compatible con el cálculo de probabilidades si se disponía de un tiempo tan amplio como el que Diderot proponía (Diderot 1992: 25 y 137). En este caso, el uso de la palabra "Naturaleza" se correspondería con la variable 13 de Lovejoy: "diversificación progresiva de los tipos en el proceso temporal, evolución continua” (Lovejoy 1952: 72). Si fuera así, estaríamos ante procesos de autoorganización, no ante diseño inteligente y dirigido.

En Consideraciones Dios es el gran ausente. Esto es significativo porque en el tema del origen del lenguaje se ha recurrido con mucha frecuencia al Dios creador. Al afrontar el tema Smith recurrió a la conocida idea de las consecuencias no previstas de nuestras acciones (Otteson 2002: 258-289), pero también a una comparación con la historia de las máquinas que remite a la variable 13 de Lovejoy, es decir, a la autoorganización natural a través de un proceso temporal largo, no de un fíat divino (Smith 2018: 92-93). No está de más recordar que en 1761 Hume ya estaba trabajando en los Diálogos sobre la religión natural, donde su alter ego Filón puso en duda la legitimidad de extender algo que el ser humano hace, a saber, diseñar cosas, a la totalidad de la realidad (Hume 1994: 84). Además, aunque Hume pensaba, como Smith, que el argumento del diseño definía la variante más noble del pensamiento religioso (Hume 2010: 35; Smith 1998: 119-121; Smith 1982c: 113-114), lo abordó como una construcción de la mente humana a lo largo de su historia mundana. No se trataba de Teología Natural sino de Historia Natural de la religión, en el caso de Smith un episodio de la historia de la Física. 
Francesco Luna afirmaba que la mano invisible era un "lapsus linguae" que solo daba a entender que algo requería una explicación mejor (Luna 1996: 131155). Aunque esa terminología nos parece demasiado extrema, creemos que interpretarla en estos términos heurísticos es lo mejor, sobre todo si se conecta con la interpretación en términos de autoorganización social desarrollada por Otto Mayr (Mayr 1986: 171-180) y por Jonathan Sheeman y Dror Wahrman (Sheeman y Wahrman 2015: 264-269). En este caso la mano invisible y las consecuencias no planificadas de nuestras acciones se referirían a procesos derivados de la conducta cooperativa lingüística que surgió para afrontar una precariedad corporal que no podíamos cancelar. Esto, además, conduce a la interpretación del "problema de Adam Smith" como un pseudoproblema. Es lo que trataremos a continuación.

\section{4. ¿El Problema de Adam Smith? Tipología y fundamentos DE LA COOPERACIÓN}

Lo visto hasta ahora nos obliga a replantearnos la visión que tenía Smith de la naturaleza emocional del vínculo social, tradicionalmente dicotomizada entre la simpatía y el egoísmo. Se trata del conocido y recurrente "problema de Adam Smith”. En 1853 Carl G. A. Knies afirmó que tras su viaje a Francia de 1764-1766 Smith cambió su manera de pensar, lo que explicaría la diferencia entre La teoría de los sentimientos morales, basada en la simpatía, y La riqueza de las naciones, basada en el egoísmo. Cuando H. T. Bucklein respondió en 1861 afirmando que, en realidad, se trataba de dos dimensiones de una misma naturaleza humana (Raphael 1989: 88-90; Raphael 2007: 115-126; Montes 2017: 40-79), surgió una polémica que, según Leónidas Montes, "continúa siendo hasta hoy motivo de debate" (Montes 2017: 79). Creemos que la opción que se elija se relaciona con la posición que se adopte en la cuestión de la mano invisible: si existe un "problema de Adam Smith", entonces la interpretación teológica de esta es aconsejable; en caso contrario, es preferible la interpretación retórica. Aquí no podemos profundizar en este debate, pero nos posicionaremos con quienes piensan, como escribió Iain McLean, que hoy "das Adam Smith-Problem ist kein Problem" porque conocemos las Lecciones sobre Jurisprudencia, donde Smith, antes de su viaje a Francia, desarrolló el argumento de la primera parte de La riqueza de las naciones (McLean 2006: 82).

Por nuestra parte, creemos que el problema se suaviza si se acepta que la noción smithiana de simpatía no fue excesivamente empática y que su mercado no fue una guerra entre egoístas. Con respecto a lo primero, en La teoría de los sentimientos morales la simpatía no era una fuerza afectiva basada en la conexión personal, 
sino un recurso para enjuiciar el comportamiento ajeno. Difería de Hume, para quien la simpatía se acercaba más a un contagio emocional (Hume 1988: 439-441). La simpatía de este producía una experiencia (Baillie 2000: 56-57), la de Smith un juicio (Raphael 2007: 116; Sagar 2017: 681-705). Refiriéndose a la existencia de un "espectro de simpatía" (aprovecharemos este concepto en lo que sigue) en el caso de Smith, Griswold, quien consideraba su obra como no problemática (Griswold 1999: 30), calificó el uso genuino del término por parte de nuestro autor como algo situacional y no emocional, interpretándolo como un recurso de objetividad que "demanda cierto grado de entendimiento" (Griswold 1999: 87).

Como vimos anteriormente (recurriendo a Condillac), la simpatía provenía de la comprensión de las necesidades corporales de los demás gracias al lenguaje (López Lloret 2019: 90-91), el mismo marco estructural que está en la base de La riqueza de las naciones, donde Smith afirmó que la división del trabajo, cuyo sentido era aumentar la producción y, con ella, el consumo, se debía a una propensión al intercambio consecuencia del lenguaje (Smith 1994: 44; Smith 1981: 25). Según pensaba, a la división del trabajo le subyacía una fórmula lingüística identificada en el Borrador de La riqueza de las naciones como "dame aquello que quiero y tendrás esto que quieres” (Smith 2017: 150; Smith 1982b: 571), lo cual se acompañó de una observación casi tan famosa como su metáfora de la mano invisible:

No es la benevolencia del carnicero, el cervecero, o el panadero lo que nos procura nuestra cena, sino el cuidado que ponen ellos en su propio beneficio. No nos dirigimos a su humanidad sino a su propio interés, y jamás les hablamos de nuestras necesidades sino de sus ventajas (Smith 1994: 46; Smith 1981: 26-27).

Griswold argumentó que el tema de este párrafo era la virtud de la prudencia, pero nosotros creemos que era la retórica (Griswold 1999: 209; Alonso-Cortés y Cabrillo 2012: 709-732). Además, no creemos que la conexión del lenguaje con el intercambio económico que propuso Smith implique que los precios sean como el lenguaje (Yaeger 1998: 15-27), sino que el mercado dependía del lenguaje (Kalyvas y Katznelson 2009: 549-580). Si en el mercado funciona la regla de las consecuencias no previstas de nuestras acciones (Hayek 1967: 96-105), conectada usualmente con la metáfora de la mano invisible, eso se debe a su base lingüística, en la que, según Otteson, tuvo su origen dicha regla (Otteson 2002: 258-289). Nosotros añadimos que esta no se basaba solo en lenguaje, sino también en su complemento, la debilidad corporal que nos obligaba a socializarnos.

Como decíamos, el párrafo trataba sobre la persuasión retórica en la demanda de carne, cerveza y pan, es decir, con las necesidades de nuestro cuerpo. En las 
sociedades comerciales seguimos hablando, pues, de nuestras carencias para lograr ayuda y ayudar. El que Smith hablase de "propio interés" ha desorientado a muchos analistas, que han detectado problemas para articular esto con su ética de la simpatía (McLean 2006: 82-85). Sin embargo, si se minimiza, como propusimos, la distancia entre la simpatía y el interés y se lee el párrafo en términos de lenguaje y necesidades corporales, el problema desaparece. Se trata, en realidad, de una gradación de tipos de colaboración, como la que Smith expuso en el siguiente párrafo de La teoría de los sentimientos morales, poco analizado en este contexto:

Cuando la ayuda necesaria es mutuamente proporcionada por el amor, la gratitud, la amistad y la estima, la sociedad florece y es feliz. Todos sus integrantes están unidos por los gratos lazos del amor y el afecto, y son por así decirlo impulsados hacia un centro común de buenos oficios mutuos (Smith 1997: 185; Smith 1982a: 85).

Este momento afectivo, para Smith el preferible, no se excluía del mundo económico, pues en La riqueza de las naciones afirmó que el comercio "debería ser entre las naciones $[\ldots]$ como entre los individuos [...] un lazo de unión y amistad" (Smith 1994: 564; Smith 1981: 493). No obstante, la interdependencia se podía desarrollar de maneras diferentes, por lo cual introdujo un segundo modelo de relación, el propio del panadero, el carnicero o el cervecero en tanto que profesionales (no necesariamente egoístas):

$[\ldots]$ aunque la asistencia necesaria no sea prestada por esos motivos tan generosos y desinteresados [...] la sociedad, aunque menos feliz y grata, no necesariamente será disuelta. La sociedad de personas distintas puede subsistir, como la de comerciantes distintos, en razón de su utilidad, sin ningún amor o afecto mutuo; y aunque en ella ninguna persona debe favor alguno o está en deuda de gratitud con nadie, la sociedad podría sostenerse a través de un intercambio mercenario de buenos oficios de acuerdo con una evaluación consensuada (Smith 1997: 185-186; Smith 1982a: 85-86).

En la tercera forma la interdependencia se rompe, pues los ciudadanos están "prestos a herir y dañar a otros", haciéndose necesaria la aplicación de la justicia (Smith 1997: 186; Smith 1982a: 86). Por lo tanto, entre el amor y la guerra se hallaba el interés, el cual, pese a no ser lo más estimado por Smith, daba más estabilidad al lazo social (esta relación es la que se estableció entre las dos personas con que se abría Consideraciones). No se trata de una contradicción sino de una gradación de posibilidades no incompatibles: el cervecero desea nuestro dinero, pero no nos odia por eso. 
Smith desarrolló otra gradación en sus Lecciones sobre Jurisprudencia. Analizando el origen de la propiedad privada recurrió al ejemplo de Locke de alguien a quien se quitara una fruta que había cogido de un árbol (Locke 1991: 224; Raphael 2007: 109-110). Esto recuerda a las escenas construidas por Condillac y por el propio Smith al comienzo de Consideraciones. La fruta era una necesidad corporal tanto para el que la cogió como para el que se la quitó, aunque solo el primero empleó su esfuerzo y su tiempo (Smith: 1995: 50; Smith 1982b: 17). A partir de aquí Smith afinó una casuística basada en tres momentos. Primero:

Si yo tuviese deseo de arrancar una manzana y hubiese estirado mi mano hacia ella, pero otro más vivo viene y la arranca antes que yo, un espectador imparcial pensará que hubo una gran infracción de las buenas maneras y la cortesía, pero no la consideraría una violación de la propiedad.

Segundo:

Si, después de que yo hubiese cogido la manzana, se me cayera y otro la atrapara, sería aún menos cívico y una afrenta grave muy cercana a una violación del derecho de propiedad.

\section{Tercero:}

Si alguien intentara arrebatarla de mi mano cuando tengo su posesión real, el circunstante estaría inmediatamente de acuerdo en que mi propiedad había sido violada, y estaría de acuerdo conmigo en recuperarla o impedir de antemano el daño, incluso suponiendo que yo usara la violencia para lograr mi propósito (Smith 1995: 51; Smith 1982b: 19).

Leída a partir de Condillac (Condillac 1999: 145), la conjetura que abre Consideraciones reflejaría el momento en el que el lenguaje, incluida la palabra ("árbol") y el gesto, instauró la conducta colaborativa del espectador, quien no se adelantó para quedarse la pieza sino para regalársela al actor, cuya precariedad corporal comprendía. Si unimos todos los registros que hemos referido en esta sección obtendremos una gradación minuciosa en las formas de cooperación: afecto, ayuda, buenas maneras, falta de civismo y agresión, en cuyo centro se halla la interacción económica. En todos los momentos, salvo los dos últimos, aparecen tanto el interés como la simpatía. Smith no plantea una contradicción entre ellos sino un proceso por el que la benevolencia afectuosa (máxima entre familiares y amigos) tiende a difuminarse cuando el número de los que interactúan crece hasta el punto en el que lo hizo en las sociedades comerciales urbanas (Pena López y Sánchez Santos 2018: 100). Aunque la fórmula lingüística del intercambio 
se mantenía, según la expresión de Smith, de forma "mercenaria" (es decir, sin vínculo personal basado en el afecto), no se trataba de algo anti o asocial, pues la necesidad cooperativa basada en la debilidad y sutilidad del cuerpo humano no se suspendía. Al contrario, se hacía evidente que cada uno de nosotros estaba "necesitado de la cooperación y ayuda de grandes multitudes" (Smith 1994: 45; Smith 1981: 26) y "de un número infinito de manos" (Smith 1995: 387; Smith 1982b: 340). Esto no hubiera sido posible si el vínculo se hubiera basado solo en la amistad y el amor.

\section{CONCLUSIONES: DEBILIDAD, NO CORRUPCIÓN}

La tesis central de este artículo es que el pensamiento ético, estético y económico de Adam Smith se basó en la conexión de la sofisticada precariedad corporal del ser humano con su capacidad para el lenguaje, una situación que no mejoró, empeoró, se redujo, se clausuró ni se clausurará con el advenimiento de la economía comercial. Esto nos conduce a preguntarnos si Smith consideró o no esta debilidad como el resultado de un proceso de corrupción o degeneración, como el efecto de una caída proveniente del pecado o, sin más, como una suerte de perversión natural. De hecho, usó en ocasiones una terminología bíblica, como cuando afirmó que el "tosco barro con el que está formado el grueso de la humanidad no puede ser labrado hasta una cumbre" de perfección ética (Smith 1997: 298; Smith 1982a: 162-163). Con todo, puesto que aceptamos que el problema de Smith es un pseudoproblema y que la mano invisible carece de elementos teológicos, pensamos que la valoración smithiana de nuestra debilidad corporal no debe interpretarse en esa clave.

En una larga nota de La teoría de los sentimientos morales Smith se refirió al ser humano como una criatura "endeble e imperfecta" (Smith 1997: 168; Smith 1982a: 77). También habló en ella del "Autor de la naturaleza" y "de los grandes fines de la naturaleza", a saber, la conservación y la propagación de nuestra especie, algo que es independiente de la fase económica en la que se esté. Para cumplir con esos fines, seguía diciendo, la naturaleza no ha confiado en nuestra razón sino en nuestro instinto, especialmente en "el hambre, la sed, la pasión que atrae a los sexos, el gusto por el placer, el rechazo del dolor", aunque también nos ha dotado de "un deseo del bienestar y la preservación de la sociedad" (Smith 1997: 168; Smith 1982a: 77). Condensó esto de una manera muy adecuada en la siguiente afirmación (que sigue a la observación de que Dios solo se ocupaba del orden en general, no del orden económico): 
Al ser humano le corresponde un distrito mucho más humilde, pero mucho más adecuado a la debilidad de sus poderes y la estrechez de su comprensión: el cuidado de su propia felicidad, de la de su familia, sus amigos, su país (Smith 1997: 423; Smith 1982a: 237).

El ser humano es débil y poco clarividente, pero, según Smith, no es un ser corrupto producto del pecado. Su debilidad no es moral sino intelectual y corporal. Ni Consideraciones ni las Lecciones sobre Jurisprudencia plantean nuestra debilidad como un resultado ni el lenguaje como un don, sino como un estado. No es algo a lo que se llegue sino algo de lo que se parte y Smith nunca plantea que, en este mundo o en otro, se vaya a superar. Explícitamente afirmó que el cuidado de lo colectivo, desde uno mismo hasta el país que se habita, corre de nuestra cuenta, pues ni Dios ni la naturaleza se ocupan de la economía ni de nuestros problemas. Lo que hacemos tiene consecuencias inesperadas que no controlamos y que pueden ser positivas o negativas, pero no son dirigidas por una teleología que nos transcienda. Por lo tanto, lo inesperado no nos exime de la responsabilidad que tenemos de afrontar nuestra debilidad corporal a partir de la persuasión, es decir, de la palabra y no de la fuerza. Y la palabra, decía Smith, estuvo con nosotros desde el principio.

Para Smith la sociedad colaborativa se inició con la comunicación de las necesidades corporales a través el lenguaje, comenzando un proceso de ayuda mutua que, al principio y dada la pequeña dimensión de los grupos, se basaba en la simpatía, es decir, la comprensión de las necesidades y la debilidad de los demás. A medida que los círculos sociales y productivos crecieron, el interés fue sustituyendo a la simpatía, estableciéndose una relación económica humanamente más neutra. Aunque la estructura de nuestra condición semiótica explicaba el desarrollo de formas económicas y sociales más complejas (no necesariamente mejores), más competitivas y basadas en las diferencias de estatus, nada de eso tendría sentido sin la peculiar condición de partida del cuerpo humano, su fascinante combinación de debilidad y capacidad de hablar. Cuando una sociedad olvida esto se endurece y deshumaniza.

Smith nunca dejó de tenerlo presente. Por eso, la lectura de su obra que lo deja de lado ha generado una interpretación sesgada, especialmente de La riqueza de las naciones. El deseo de que la economía sea una ciencia objetiva y predictiva como la física o la astronomía, con sus métodos inductivos y matemáticos, deja de lado a los sujetos concretos de cuya interacción surge, buscando estructuras anónimas y anómicas. Las personas, sin embargo, no son planetas que se puedan reducir a la abstracción de un punto en una elipse o de una variable en una fórmula (Montes Lira 2017: 181-182), algo que eliminaría toda consecuencia inesperada. Ante todo, 
porque "en el vasto tablero de la sociedad humana cada pieza posee un principio motriz propio, totalmente independiente" (Smith 1997: 418; Smith 1982a: 234), pero también porque este movimiento independiente es algo concreto, individual y fisiológico que, a través del lenguaje, afrontamos de una manera creativa y persuasiva, reconociendo como propias la debilidad y la necesidad de los demás.

El deseo de afrontar la economía como algo que nos pasa y no como algo que hacemos a partir de nuestra debilidad, así como la obsesión por garantizar un crecimiento económico autónomo (Hühn 2017: 2-5), olvidan esto, sin lo cual La riqueza de las naciones pierde parte de su significado. La magna obra de Smith debe leerse teniendo en cuenta su producción anterior y como parte del proyecto global unificado que, como dijimos en la Introducción, no completó (Ross 2004: 40-59). En Consideraciones y en las Lecciones sobre Jurisprudencia expuso la necesidad de reconocer la precariedad del otro como propia, estableciendo la sociedad colaborativa, y en La teoría de los sentimientos morales explicó cómo podíamos ponernos en el pellejo de esas otras personas gracias a la imaginación (Frazer 2010: 97-101; Marshall 1986: 170-171; Haakonssen 1981: 51). A pesar de la complejidad cultural, social y semiótica de las sociedades comerciales que hemos ido configurando, no hemos podido cancelar, de momento, la debilidad y la sutilidad de nuestro cuerpo, de manera que la extensión de la cooperación sigue siendo lo mejor para todos. Esto no quiere decir, en términos smithianos, que sea necesario amar a todo el mundo o ser un admirable altruista, pero tampoco lo contrario. Se trataba de opciones personales y graduales definidas en el seno de los grupos sociales de diversa extensión que construimos con nuestras interacciones. Lo que no construimos es nuestro cuerpo necesitado, cuya precariedad se renueva a cada momento. Por eso, aunque la sociedad ha pasado de las necesidades de la caza a las superficialidades del mundo comercial, basadas en el estatus y la acumulación, las demandas de nuestro cuerpo son las mismas. Nuestra piel sigue siendo delicada y sigue precisando respuestas colaborativas, constructivas y provisionales, es decir, históricamente abiertas.

Smith enseñó a sus alumnos y a sus lectores, incluidos los del siglo XXI, que el ser humano se hallaba entre las otras especies animales (cuya piel no era tan delicada y, por eso, no precisaban de una inteligencia sutil ni de una comunicación lingüística) y los dioses (que, habiendo trascendido el cuerpo, no necesitaban hablar). Enseñó que nuestro destino era colaborativo y que el ser humano tenía que definir, a través de la persuasión (es decir, del lenguaje y no de la fuerza), sus formas de socialización, incluidas las económicas. No fue el profeta de un mercado salvaje que estuviera a medio camino del sistema solar newtoniano y de la selva 
evolutiva spenceriana, sino un ilustrado que comprendió la necesidad que tenemos de hablar, acordar y pactar con los demás a causa de nuestra debilidad. Nos enseñó que tanto los individuos como las naciones se necesitan entre sí.

\author{
Jorge López, Lloret \\ Universidad de Sevilla \\ lopezlloret@us.es
}

\title{
BibliografíA
}

Alonso-Cortés, A. y CABrillo, F. (2012): "From merchants to speakers: The common origins of trade and language", European Journal of History of Economic Thought, no 19/5, pp. 709-732.

AsHelford, J. (1996): The Art of Dress. Clothes through History 1500-1914, London: National Trust Books.

Bascones, L. M. y Domínguez, M. (2001): "Palabras, monedas y seres vivos. Adam Smith y la historia conjetural del origen de la lengua", Política y Sociedad, n 37, 57-79.

BAiLliE, J. (2000): Hume on Morality, London: Routledge.

BuRY, J. (2009): La idea del progreso, Madrid, Alianza.

CiCERÓN (1997): La invención retórica, Madrid: Tecnos.

Condillac, E. B. de (1999): Ensayo sobre el origen de los conocimientos humanos, Madrid: Tecnos.

Conlin, J. (2016): Adam Smith, London: Reaktion Books.

Diderot, D. (1992): Sobre la interpretación de la naturaleza, Barcelona: Anthropos.

Diderot, D. (2011): Tratado de la barbarie de los pueblos civilizados, Barcelona: Pasado y Presente.

Diderot, D. (2013): Suplemento al viaje de Bougainville, Barcelona: Sd-edicions.

Diderot, D. (2016): El paseo del escéptico, Pamplona: Laetoli.

Diderot, D. y D’Alembert, J. le R. (1751): Encyclopédie, Tome V, París: Briasson, David, Le Breton, Durand.

Douglass, R. (2017): "Morality and Sociability in Commercial Society: Smith, Rousseau and Mandeville", The Review of Politics, $\mathrm{n}^{\circ}$ 79, pp. 597-620.

Fleischacker, S. (2005): On Adam Smith's Wealth of Nations, Princeton/Nueva Jersey: Princeton University Press.

Frazer, M. L. (2010): The Enlightenment of Sympathy, Oxford: Oxford University Press.

Griswold, Ch. L. (1999): Adam Smith and the Virtues of Enlightenment, Cambridge: Cambridge University Press.

Griswold, Ch. L. (2018): Jean-Jacques Rousseau and Adam Smith. A Philosophical Encounter, Abingdon: Routledge.

HaAkONSSEN, K. (1981): The Science of a Legislator. The Natural Jurisprudence of David Hume and Adam Smith, New York: Cambridge University Press.

HayeK, F. A. (1967): Studies in Philosophy, Politics and Economics, London: Routledge. 
HüHN, M. P. (2019): “Adam Smith’s Philosophy of Science: Economics as Moral Imagination", Journal of Business Ethics, $\mathrm{n}^{\circ} 155$, pp. 1-15.

Hume, D. (1988): Tratado de la naturaleza humana (1739-40), Madrid: Tecnos.

HumE, D. (1994): Diálogos sobre la religión natural (1779), Madrid: Tecnos.

Hume, D. (2010): Historia natural de la religión (1757), Madrid: Tecnos.

Hutcheson, F. (1750): Reflections upon Laughter, and Remarks upon The Fable of the Bees, Glasgow: R. Urie.

Hutcheson, F. (2002): An Essay on the Nature and Conduct of the Passions and Affections, Indianapolis: Liberty Fund.

Hutcheson, F. (2006): Logic, Metaphysics, and the Natural Sociability of Mankind, Indianapolis: Liberty Fund.

Hutcheson, F. (2007): Philosophiae Moralis Institutio Compendia, Indianapolis: Liberty Fund.

Hutcheson, F. (2008): An Inquiry into the Original of Our Ideas of Beauty and Virtue, Indianapolis: Liberty Fund.

Jantzen, B. C. (2014): An Introduction to Design Arguments, Cambridge: Cambridge University Press.

Kalyvas, A. y Katznelson, I. (2009): "The rhetoric of the market. Adam Smith on recognition, speech, and exchange", The Review of Politics, n 63/3, pp. 549-580.

Kennedy, G. (2009): "Adam Smith and the Invisible Hand: From Metaphor to Myth", Econ Journal Watch, no 6/2, pp. 239-263.

Kennedy, G. (2011): "Adam Smith and the role of the metaphor of an invisible hand", Economic Affairs, no 31, pp. 53-57.

Kennedy, G. (2017): An Authentic Account of Adam Smith, Cham: Palgrave Macmillan.

LAUGIER, M.-A. (1999): Ensayo sobre la arquitectura, Madrid: Akal.

LOCKE, J. (1991): Dos ensayos sobre el gobierno civil, Madrid: Espasa Calpe.

López Lloret, J. (2019): "El fundamento lingüístico del pensamiento de Adam Smith", Revista Empresa y Humanismo, Vol. XXII/1, pp. 71-100.

Lovejoy, A. O. (1948): Essays in the History of Ideas, Baltimore: The John Hopkins.

LunA, F. (1996): "From the History of Astronomy to the Wealth of Nations: Wonderful Wheels and Invisible Hands in Adam Smith's Major Works", en Vaz, D. y Velupillai, K. (eds.), Inflation, Institutions and Information, London: Macmillan, pp. 131-155.

Mankin, R. (2008): "Pins and needles: Adam Smith and the sources of the Encyclopédie", The Adam Smith Review, no 4, pp. 181-205.

Marshall, D. (1986): The Figure of Theater. Shaftesbury, Defoe, Adam Smith, and George Eliot, New York: Columbia University Press.

MaYr, O. (1986): Authority, Liberty and Automatic Machinery in Early Modern Europe, Baltimore: The Johns Hopkins University Press.

MCLEAn, I. (2006): Adam Smith. Radical and Egalitarian, Edinburgh: Edinburgh University Press, 2006).

Montes Lira, L. (2017): Adam Smith en Contexto, Madrid: Tecnos. 
Oslington, P. (2014): "Divine Action, Providence and Adam Smith’s Invisible Hand", en Oslington, P. (ed.): Adam Smith as Theologian, New York: Routledge, pp. 61-74.

Otteson, J. R. (2002): Adam Smith's Marketplace of Life, Cambridge: Cambridge University Press.

Pena López, J. A. y Sánchez Santos, J. M. (2007): "El problema de Smith y la relación entre moral y economía”, Isegoría, no 36, pp. 81-103.

Raphael, D. D. (1989): Adam Smith, Oxford: Oxford University Press.

RApHAEL, D. D. (2007): The Impartial Spectator, Oxford: Oxford University Press.

Rasmussen, D. C. (2008): The Problems and Promise of Commercial Society. Adam Smith's Response to Rousseau, University Park/Pennsylvania: The Pennsylvania State University Press.

Roberts, R. (2015): Cómo Adam Smith puede cambiar tu vida, Barcelona: Antoni Bosch.

Ross, I. S. (2004): “'Great works upon the anvil' in 1785. Adam Smith's Projected Corpus of Philosophy", The Adam Smith Review, no 1, pp. 40-59.

Rousseau, J.-J. (1989): Discurso sobre el origen y los fundamentos de la desigualdad entre los hombres y otros escritos, Madrid: Tecnos.

Rousseau, J.-J. (2001): Discurso sobre la economía politica, Madrid: Tecnos.

SAGAR, P. (2017): “Beyond sympathy: Smith's rejection of Hume's moral theory", British Journal for the History of Philosophy, $\mathrm{n}^{\circ}$ 25/4, pp. 681-705.

Samuels, W. J. (2014): Erasing the Invisible Hand. Essays on an Elusive and Misused Concept in Economics, Cambridge: Cambridge University Press.

SCHLIESSER, E. (2017): Adam Smith. Systematic Philosopher and Public Thinker, Oxford: Oxford University Press.

SEIDEL, W. (1989): "Zählt die Musik zu den imitativen Künsten? Zur Revision der Nachahmungsästhetik durch Adam Smith", en J. P. Fricke (ed.), Die Sprache der Musik, Regensburg: Gustav Bosse Verlag, pp. 495-511.

Sheeman, J. y Wahrman, D. (2015): Invisible Hands. Self-Organization and the Eighteenth Century, Chicago: The University of Chicago Press.

Smith, A. (1981): An Inquiry into the Nature and Causes of the Wealth of Nations, Indianapolis: Liberty Fund.

Smith, A. (1982a): The Theory of Moral Sentiments, Indianapolis: Liberty Fund.

SмITH, A. (1982b): Lectures on Jurisprudence, Indianapolis: Liberty Fund.

Smith, A. (1982c): Essays on Philosophical Subjects, Indianapolis: Liberty Fund.

Smith, A. (1985): Lectures on Rhetoric and Belles Lettres, Indianapolis: Liberty Fund.

Sмiтh, A. (1994): La riqueza de las naciones, Madrid: Alianza.

SмITH, A. (1995): Lecciones sobre Jurisprudencia, Granada: Comares.

Smith, A. (1997): La teoría de los sentimientos morales, Madrid: Alianza.

Sмith, A. (1998): Ensayos filosóficos, Madrid: Pirámide.

Smith, A. (2017): Escritos preliminares de La riqueza de las naciones y Consideraciones sobre la primera formación de las lenguas, Madrid: Biblioteca Nueva.

SmITH, A. (2018): Consideraciones sobre la formación original de los lenguajes, Oviedo: KRK. 
VINER, J. (1965): “Guide to John Rae's Life of Adam Smith", en J. Rae, Life of Adam Smith, New York: Augustus M. Kelley.

VivenzA, G. (2001): Adam Smith and the Classics, Oxford: Oxford University Press.

Wells, G. A. (1987): The Origin of Language. Aspects of the Discussion from Condillac to Wundt, La Salle/Illinois: Open Court.

Willey, B. (1950): The Eighteenth-Century Background, London: Chato and Windus.

YEAGER, V. L. B. (1998): “Are markets like language?”, The Quarterly Journal of Austrian Economics, n $1 / 3$, pp. 15-27. 